\title{
AFB POSITIVITY IN TUBERCULAR LYMPHADENITIS AND ITS CORRELATION WITH CYTOLOGICAL PATTERNS IN A TERTIARY CARE CENTRE IN SAHARANPUR
}

\author{
Neetu Goyal1, Rachna Sharma², Somya Jain ${ }^{3}$
}

${ }_{1}^{1}$ Assistant Professor, Department of Pathology, SMMH, Government Medical College, Saharanpur, Uttar Pradesh, India. ${ }^{2}$ Assistant Professor, Department of Pathology, SMMH, Government Medical College, Saharanpur, Uttar Pradesh, India. ${ }^{3}$ Consulting Physician, Department of Medicine, AKJ Healthcare and Diagnostics, Saharanpur, Uttar Pradesh, India.

\section{ABSTRACT}

\section{BACKGROUND}

Tuberculosis is one of the most common causes of lymphadenopathy in India. It is possible to diagnose it with the help of a minimally invasive procedure known as Fine-Needle Aspiration Cytology (FNAC) and thereby unnecessary surgical interventions can be avoided.

Aim-

The aim of the present study was to study the cytological patterns of tuberculous lymphadenitis and to correlate the Acid-Fast Bacilli (AFB) positivity with cytological patterns and also to find out overall AFB positivity.

Objectives-

1. To study the cytological patterns of tubercular lymphadenitis.

2. To correlate the cytological pattern with AFB positivity.

3. To find out overall AFB positivity in cytology of tubercular lymphadenitis.

\section{MATERIALS AND METHODS}

This is a descriptive study. We studied a total of 180 cases of tuberculous lymphadenitis. Cases were retrieved and analysed retrospectively between 2015 and 2018 for three different cytological patterns A (Epithelioid granuloma without necrosis), B (Epithelioid granuloma with necrosis) and C (Necrosis without epithelioid granuloma). Ziehl-Neelsen stain (ZN) for AFB was done in all cases.

\section{RESULTS}

Patterns A, B and C were observed in 36 (20\%), 93 (51.6\%) and 51 (28.4\%) cases, respectively. AFB positivity was found in 1 $(2.7 \%)$ case of pattern A, $60(65 \%)$ cases of pattern B and $45(88.2 \%)$ cases of pattern C. The overall AFB positivity was seen in $58.88 \%(106 / 180)$ cases.

\section{CONCLUSION}

FNAC is a minimally invasive, sensitive and safe procedure to diagnose tuberculous lymphadenitis. Study of both cytological patterns and ZN staining for AFB can improve the diagnostic yield. Whether or not granuloma is present, ZN stain must be employed whenever infective pathology is suspected.

\section{KEY WORDS}

Cytological Patterns, Fine Needle Aspiration Cytology, Tuberculous Lymphadenitis, Ziehl-Neelsen Stain.

HOW TO CITE THIS ARTICLE: Goyal N, Sharma R, Jain S. AFB positivity in tubercular lymphadenitis and its correlation with cytological patterns in a tertiary care centre in Saharanpur. J. Evolution Med. Dent. Sci. 2018;7(30):3365-3368, D0I: $10.14260 /$ jemds/2018/759

\section{BACKGROUND}

Tuberculosis continues to be the biggest health problem in developing countries with enormous social and economic implications. India has the highest burden of tuberculosis in the world, an estimated 2 million cases annually and accounts for approximately one-fifth of the global incidence.[1],[3] In India, 1000 people a day or one per minute die of tuberculosis.[2]

Since the mid-1980s, both in developing and developed countries the increasing incidence of extrapulmonary TB has

'Financial or Other Competing Interest': None.

Submission 11-06-2018, Peer Review 07-07-2018,

Acceptance 14-07-2018, Published 23-07-2018.

Corresponding Author:

Dr. Somya Jain,

Court Road, Opposite ITO,

Behind Petrol Pump, Saharanpur,

Uttar Pradesh, India.

E-mail: drsomya21@gmail.com

DOI: $10.14260 /$ jemds $/ 2018 / 759$

(c) (1) $(9)$ been noted. ${ }^{[4]}$ In India, extrapulmonary TB accounting 20\% of all TB cases and its prevalence in the country varies between $8.3 \%$ and $13.1 \%$ in different districts according to cohort analysis by the Central TB Division, Ministry of Health and Family Welfare in 2002.[5] In India and other developing countries, TB lymphadenitis is the most common form of extrapulmonary TB and it comprises $35 \%$ of cases.[6] Infection with the Human Immunodeficiency Virus/ Acquired Immunodeficiency Syndrome (HIV/AIDS) is associated with an increased frequency of both pulmonary and extrapulmonary TB, especially lymphadenitis. $[7,8]$

TB lymphadenitis is usually more common in females and in younger age groups in contrast to pulmonary $\mathrm{TB}$, which is more common in males and in the older age group and a peak age of onset of 20 - 40 years. $[9,10]$ There are various diagnostic modalities available for the diagnosis of TB lymphadenitis like Fine-Needle Aspiration Cytology (FNAC), histopathological examination excised lymph nodes, culture, Ziehl-Neelsen(ZN) staining for Acid Fast Bacilli (AFB), imaging studies and molecular tests. Mycobacterial culture being a gold standard method could be useful as a definitive 
diagnosis. Even though culture is considered as gold standard for the diagnosis, FNAC should be used as the initial diagnostic test in suspected cases of $\mathrm{TB}$, as it is a simple minimally invasive, less expensive procedure with excellent sensitivity and specificity.[11] The present study was undertaken to evaluate cytological patterns of tuberculous lymphadenitis, to correlate the AFB positivity with cytomorphological patterns and also to find out overall AFB positivity.

\section{MATERIALS AND METHODS}

\section{Study Design}

The present descriptive study was undertaken at Department of Pathology in our institution, a tertiary care centre, conducted over the period of 3 years from March 2015 to March 2018.

\section{Study Population}

A total of 180 cases with cytologically proven tuberculous lymphadenitis were included in the present study. Patients with non-tuberculous mycobacterial infections such as Mycobacterium scrofulaceum, Mycobacterium aviumintracellulare complex and Mycobacterium kansasii, Pulmonary Koch's, Bacillus Calmette-Guerin vaccination causing lymphadenitis and periodic acid-Schiff positive organisms in FNAC smears were also excluded from this study. Non-tuberculous mycobacterial infections were excluded by identification of only microabscesses, ill-defined granulomas, non-caseating granulomas and a small number of giant cells[12] and Pulmonary Koch's, Bacillus CalmetteGuerin vaccination causing lymphadenitis were excluded by detailed clinical history collected from medical records.

\section{Collection of Data}

Clinical data such as site of aspiration, number of lymph nodes involved, nature of aspirates (blood mixed material, purulent/ pus, caseous or cheesy) were obtained from patient's medical records.

\section{Cytological Evaluation}

May-Grunwald Giemsa and Haematoxylin and Eosin stained smears were studied for pattern analysis. According to the Das et al study,[13] cytology smears revealing cytomorphological features of tuberculous lymphadenitis were grouped into three categories: Pattern A: Epithelioid granuloma without necrosis [Figure 1a]; Pattern B: Epithelioid granuloma with necrosis [Figure 1b]; and Pattern C: Necrosis without epithelioid granuloma [Figure 1c]. Figure $1 \mathrm{~d}$ shows acid fast bacilli on ZN stain.[14]

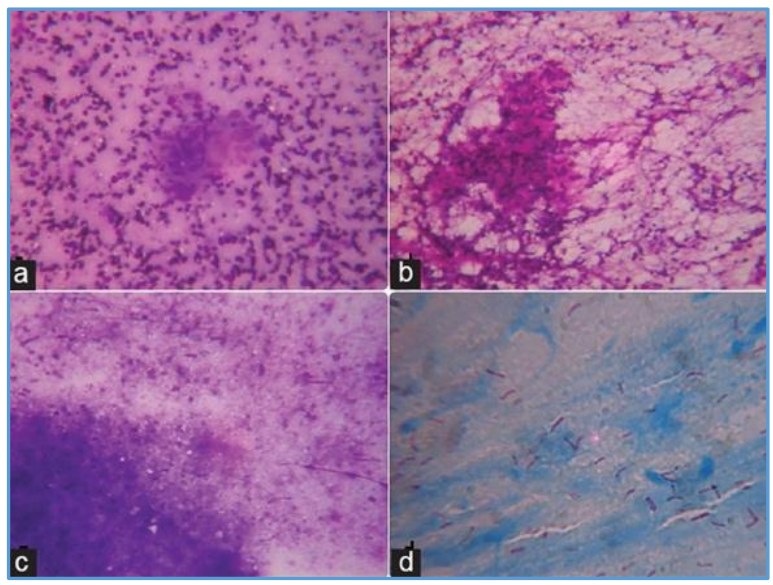

Figure 1a, 1b, 1c, 1d

\section{Statistical Analysis}

All the statistical analysis was performed using IBM SPSS Statistics for Windows (version 20.0, IBM Corporation, Armonk, New York, USA). Correlation between various cytomorphological patterns and AFB positivity was assessed using Chi-square test. The $\mathrm{p} \leq 0.05$ was considered for statistical significance.

\section{RESULTS}

Out of 180 cases, 106 were AFB positive cases and remaining 74 was AFB negative, but showed cytological features of tuberculous lymphadenitis.

\begin{tabular}{|c|c|c|c|c|c|}
\hline Age & $\begin{array}{c}<\mathbf{5} \\
\text { Years }\end{array}$ & $\begin{array}{c}\mathbf{5 - 1 0} \\
\text { Years }\end{array}$ & $\begin{array}{c}\mathbf{1 0 - 2 0} \\
\text { Years }\end{array}$ & $\begin{array}{c}\mathbf{2 0 - 4 0} \\
\text { Years }\end{array}$ & $\begin{array}{c}>40 \\
\text { Years }\end{array}$ \\
\hline Male & 2 & 3 & 4 & 46 & 10 \\
\hline Female & 0 & 1 & 5 & 80 & 20 \\
\hline \multicolumn{7}{|c|}{ Table 1. Age and Gender Wise distribution of patients } \\
included in the Study
\end{tabular}

Site and Number of Lymph Node Involvement

In the present study, the most common site of involved lymph nodes was cervical lymph nodes in 164 (91.1\%) followed by axillary lymph nodes in 14 (7.7\%) and inguinal lymph nodes in $2(1.1 \%)$ cases. The most common presentation was single palpable cervical lymphadenopathy in 126 (70\%) cases followed by multiple unilateral palpable cervical lymphadenopathy in $45(25 \%)$ and multiple bilateral cervical lymphadenopathy in $9(5 \%)$ cases.

\section{Nature of Aspirate}

On the basis of appearance of aspirate, blood mixed aspirate were noted more commonly in $108(60 \%)$ cases followed by purulent/ pus material in $45(25 \%)$ cases and caseous or cheesy material in 27 (15\%) cases. Blood mixed aspirate was predominantly seen in Pattern A and B and purulent/ pus and caseous or cheesy material were predominantly seen in Pattern C.

\section{Cytological Pattern, Acid-Fast Bacilli Positivity}

Of these 180 cases Pattern A was observed in 36 (20\%) cases, Pattern B was observed in $93(51.6 \%)$ cases and Pattern C was observed in $51(28.4 \%)$ cases. AFB positivity was found in $1(2.7 \%)$ case of Pattern A, $60(65 \%)$ cases of Pattern B and $45(88.2 \%)$ cases of Pattern C. The highest percentage of AFB positivity $(64.7 \%)$ was observed in aspirate containing purulent/ pus and caseous/ cheesy material. The overall AFB positivity was seen in $58.8 \%(106 / 180)$ cases.

The present study showed significant statistical difference between AFB positivity and cytomorphological patterns $(\mathrm{p}<0.0001)$.

\begin{tabular}{|c|c|}
\hline Cytological Pattern & AFB Positivity \\
\hline Pattern A & $2.7 \%$ \\
\hline Pattern B & $65 \%$ \\
\hline Pattern C & $88.2 \%$ \\
\hline Table 2. Correlation of Cytological Pattern with AFB \\
Positivity \\
\hline
\end{tabular}

\section{DISCUSSION}

On clinical examination, cervical lymph nodes (91.1\%) were more commonly involved and inguinal lymph nodes were 
least commonly involved in the present study similar to most of the recently published studies.[15,16,17,18] This is because the organism usually gains access to the cervical lymph nodes through the tonsillar lymphoid tissue.

In this study, most common presentation was single palpable cervical lymphadenopathy in $70 \%$ cases, lower as compared to Chand et al (81.6\%) study[16] and higher than Nidhi et al (63.3\%) study.[15] We observed multiple unilateral palpable cervical lymphadenopathy in $25 \%$ cases and multiple bilateral cervical lymphadenopathy in 5\% cases, which were almost comparable to Nidhi et al study.[15] Due to various initiatives by governmental and non-governmental agencies, the awareness regarding ТВ has been increased. This might explain the high number of cases presenting with single lymph node enlargement.

According to gross nature of aspirate, blood mixed aspirates were noted in 60 followed by purulent/ pus material in $25 \%$ and caseous or cheesy material in $15 \%$ cases in the current study, whereas Hemalatha et al[19] observed blood mixed aspirates in $87.3 \%$ and purulent to cheesy materials in $12.7 \%$ cases. Pattern A and B were predominantly observed in blood mixed aspirate, whereas Pattern C was predominantly observed in purulent/ pus and caseous or cheesy aspirates in the present study similar to Hemalatha et al study.[19]

Cytological pictures of tuberculous lymphadenitis was described by various authors with minor modifications with different names, viz. patterns or category.[19,20] We described cytological pictures in our study as patterns. In our study, most common cytomorphological pattern observed was Pattern B (51.6\%) comparable to Hemalatha et al and Gupta et al studies $[19,21]$ and it was higher than studies done by Nidhi et al[15] and Chand et al[16] (16.4\% and $21.8 \%$, respectively). The formation of granuloma is dependent on a fairly good immune system within the host. In immunocompromised situations, the immunological interplay between lymphocytes, macrophages, the organism and various interleukins/ cytokines may not be efficient to result in granuloma formation. Hence, when AFB positivity is observed without granuloma formation, other comorbid immunodeficient states should be thought of.

Similar to few Indian studies, $[13,15,19]$ we observed more number of AFB positivity in Pattern C (88.2\%) followed by Pattern B (65\%) in the current study. Pattern A showed 2.7\% AFB positivity in our study, whereas Nidhi et al[15] and Chand et al[16] studies noted $3.25 \%$ and $2.56 \%$ AFB positivity, respectively. We found maximum percentage of AFB positivity (64.7\%) in aspirate containing purulent/ pus and caseous/ cheesy material similar to studies done by various authors. $[15,19,22]$ If the purulent material is aspirated, slides should be stained for ZN staining to rule out tuberculous lymphadenitis, because it can mimic as acute suppurative lesions.[19] The present study showed overall AFB positivity in 58.8\% cases comparable to most of the published studies ${ }^{[16,17,19]}$ and lower than Nidhi et al[15] and Ergete and Bekele[20] studies. Varying AFB positivity has been found by many authors ranging from $35.6 \%$ to $55.2 \%$.[19] Slides can be decolourised for ZN staining if cytological features of TB and necrosis present. This may increase the AFB positivity.[15] Yield of AFB positivity can be further increased by doing repeat FNAC of a lymph node.[23] Clinical correlation and microbiological assessment should be done in AFB negative cases showing only epithelioid granulomas without necrosis. Microbiological assessment is necessary in AFB negative cases to confirm the diagnosis of $\mathrm{TB}$ as approximately $10,000-100,000$ mycobacterial organism/ $\mathrm{mL}$ of the sample should be present for smear AFB positivity.[15]

There was significant statistical difference between AFB positivity and cytomorphological patterns ( $p<0.0001$ ) similar to Hemalatha et al study.[19] Comparison of patterns of tuberculous lymphadenitis and overall AFB positivity in the present study with other studies is as shown in Table 3.

\begin{tabular}{|c|c|c|c|c|}
\hline Study & $\begin{array}{c}\text { Pattern } \\
\text { A }\end{array}$ & $\begin{array}{c}\text { Pattern } \\
\text { B }\end{array}$ & $\begin{array}{c}\text { Pattern } \\
\text { C }\end{array}$ & $\begin{array}{c}\text { Overall } \\
\text { AFB } \\
\text { Positivity }\end{array}$ \\
\hline $\begin{array}{c}\text { Ergete and } \\
\text { Bekele[20] }\end{array}$ & $3.4 \%$ & $63.7 \%$ & $32.7 \%$ & $71.7 \%$ \\
\hline Nidhi et al[15] & $14.3 \%$ & $16.4 \%$ & $39.2 \%$ & $71 \%$ \\
\hline Chand et al[16] & $28.4 \%$ & $21.8 \%$ & $15.4 \%$ & $44.54 \%$ \\
\hline $\begin{array}{c}\text { Hemalatha et } \\
\text { al[19] }\end{array}$ & $19.3 \%$ & $56 \%$ & $22.7 \%$ & $54 \%$ \\
\hline $\begin{array}{c}\text { Present Study } \\
\text { Nent }\end{array}$ & $20 \%$ & $51.6 \%$ & $28.4 \%$ & $58.8 \%$ \\
\hline
\end{tabular}

\section{CONCLUSION}

FNAC is a simple minimally invasive, cost effective and safe procedure which has excellent sensitivity and specificity to diagnose tuberculous lymphadenitis. Study of both cytomorphological patterns and ZN staining for AFB can improve the diagnostic yield. Regardless of the presence of granuloma, ZN stain must be employed whenever infective pathology is suspected.

\section{REFERENCES}

[1] World Health Organisation Global TB control report, Geneva, Switzerland: WHO.

[2] Chauhan LS. Challenges for the RNTCP in India. J Indian Med Assoc 2003;101(3):152-3.

[3] New Delhi: Central TB Division, Directorate General of Health Services, Ministry of Health and Family Welfare, 2009. Ministry of Health and Family Welfare. RNTCP Status Report. TB India 2009.

[4] Peto HM, Pratt RH, Harrington TA, et al. Epidemiology of extrapulmonary tuberculosis in the United States, 1993-2006. Clin Infect Dis 2009;49(9):1350-7.

[5] Arora VK, Chopra KK. Extra pulmonary tuberculosis. Indian J Tuberc 2007;54(4):165-7.

[6] Sharma SK, Mohan A. Extrapulmonary tuberculosis. Indian J Med Res 2004;120(4):316-53.

[7] Aguado JM, Castrillo JM. Lymphadenitis as a characteristic manifestation of disseminated tuberculosis in intravenous drug abusers infected with human immunodeficiency virus. J Infect 1987;14:1913.

[8] Finfer M, Perchick A, Burstein DE. Fine needle aspiration biopsy diagnosis of tuberculous lymphadenitis in patients with and without the acquired immune deficiency syndrome. Acta Cytol 1991;35(3):325-32. 
[9] Shubha AB, Sapna H, Dinesh RB. Tuberculosis lymphadenitis presenting a diagnostic dilemma - a case report. Int J Dent Clin 2010;2:48-52.

[10] Golden MP, Vikram HR. Extrapulmonary tuberculosis: an overview. Am Fam Physician 2005;72(9):1761-8.

[11] Handa U, Mundi I, Mohan S. Nodal tuberculosis revisited: a review. J Infect Dev Ctries 2012;6(1):6-12.

[12] Kraus M, Benharroch D, Kaplan D, et al. Mycobacterial cervical lymphadenitis: the histological features of non-tuberculous mycobacterial infection. Histopathology 1999;35(6):534-8.

[13] Das DK, Pant JN, Chachra KL, et al. Tuberculous lymphadenitis: correlation of cellular components and necrosis in lymph-node aspirate with A.F.B. positivity and bacillary count. Indian $\mathrm{J}$ Pathol Microbiol 1990;33(1):1-10.

[14] Kumar S, Ferns S, Sujatha S, et al. Acid-fast staining patterns and their correlation with HIV positivity. Acta Cytol 2005;49(1):111-2.

[15] Nidhi P, Sapna T, Shalini M, et al. FNAC in tuberculous lymphadenitis: experience from a tertiary level referral centre. Indian J Tuberc 2011;58(3):102-7.

[16] Chand P, Dogra R, Chauhan N, et al. Cytopathological pattern of tubercular lymphadenopathy on FNAC: analysis of 550 consecutive cases. J Clin Diagn Res 2014;8(9):FC16-9.
[17] Bezabih M, Mariam DW, Selassie SG. Fine needle aspiration cytology of suspected tuberculous lymphadenitis. Cytopathology 2002;13(5):284-90.

[18] Sharma S, Sarin R, Khalid UK, et al. Clinical profile and treatment outcome of tuberculous lymphadenitis in children using DOTS strategy. Indian J Tuberc 2010;57(1):4-11.

[19] Hemalatha A, Shruti P, Kumar MU, et al. Cytomorphological patterns of tubercular lymphadenitis revisited. Ann Med Health Sci Res 2014;4(3):393-6.

[20] Ergete W, Bekele A. Acid fast bacilli in aspiration smears from tuberculous patients. Ethiop J Health Dev 2000;14(1):99-104.

[21] Gupta AK, Nayar M, Chandra M. Critical appraisal of fine needle aspiration cytology in tuberculous lymphadenitis. Acta Cytol 1992;36(3):391-4.

[22] Ahmad SS, Akhtar S, Akhtar K, et al. Incidence of tuberculosis from study of fine needle aspiration cytology in lymphadenopathy and acid fast staining. Indian J Community Med 2005;30(2):63-5.

[23] Kumar N, Jain S, Murthy NS. Utility of repeat fine needle aspiration in acute suppurative lesions. Followup of 263 cases. Acta Cytol 2004;48(3):337-40. 